\title{
IDEOLOGIA, UTOPIA E CRISI DELLA POLITICA
}

\author{
GUIDO MONTANI (*)
}

Nota presentata dal m.e. Silvio Beretta

(Adunanza del 28 novembre 2019)

SuNTO. - I partiti politici che si ispirano ai valori del liberalismo, della democrazia e del socialismo non riescono più a concepire un progetto di lungo periodo per i propri concittadini e per l'umanità. Dopo il crollo del Muro di Berlino, grandi e piccole potenze hanno avviato una sorda lotta per la supremazia mondiale, alimentando conflitti locali e globali, e il ritorno del nazionalismo come ideologia dominante. In questo saggio si intende mostrare che la tesi sulla «fine delle ideologie» è infondata: le ideologie tradizionali sono incapaci di progettare un futuro di progresso perché subiscono passivamente l'ideologia della sovranità assoluta degli stati e della guerra giusta per difendere gli interessi nazionali. Il futuro dell'umanità è minacciato da una nuova corsa agli armamenti nucleari e convenzionali, ai quali i governi dedicano immense risorse, che dovrebbero invece servire per salvare il Pianeta dal surriscaldamento climatico e dallo sterminio della vita animale e vegetale. La via intrapresa dai popoli europei, con la costruzione della prima Unione sovranazionale della storia, dovrebbe ispirare anche le politiche necessarie per la costruzione di un ordine mondiale fondato sulla cooperazione pacifica tra stati e l'avvio di politiche che si propongano di consentire ai cittadini del mondo di godere dei medesimi diritti di libertà e solidarietà che, seppure imperfettamente, si sono realizzati in Europa. Il progresso dell'umanità è un'utopia positiva che può diventare realtà.

$* * *$

ABSTRACT. - Political parties inspired by the values of liberalism, democracy and socialism no longer appear to be capable of conceiving long-term projects for their fellow citizens and humanity. After the fall of the Berlin Wall, large and small powers entered into a sordid struggle for world supremacy, sparking both local and global conflicts, and heralding the return of nationalism as a dominant ideology. This essay sets out to demonstrate that the posited "end of ideology" is groundless: traditional ideologies are unable to envisage a future of progress because they passively submit to the ideology of

(*) Professore di International Political Economy all'Università di Pavia, Italy. E-mail: guido.montani23@gmail.com 
the absolute sovereignty of states and the just war to defend national interests. The future of humanity is threatened by a new arms race - regarding both nuclear and conventional weapons - to which governments are dedicating vast amounts of money; money which should actually be invested in saving the planet from global warming and the sixth extinction of animal species now under way. The path taken by the people of Europe, with the construction of the first supranational Union in history, should be a source of inspiration for formulating the policies necessary to construct a world order based on peaceful cooperation between states and to ensure that all citizens of the world enjoy the same rights of freedom and solidarity that, albeit imperfectly, have been achieved in Europe. Human progress is a utopia that can become a reality.

La recente pubblicazione del mio libro (Montani 2019a) ha rappresentato l'occasione per l'invito rivoltomi dall'Istituto Lombardo Accademia di Scienze e Lettere a svolgere una relazione a un'Adunanza. Dato il tempo a disposizione, tuttavia, non cercherò di inoltrarmi in una presentazione dei contenuti specifici trattati nei quattro capitoli del libro. Vorrei limitarmi a una esposizione dell'approccio metodologico che ho adottato nel corso delle mie ultime ricerche sul complesso problema dei rapporti tra l'ideologia e le scienze sociali. La principale difficoltà è quella di comprendere perché il concetto di ideologia sia così poco studiato dai cultori di scienze sociali, nonostante che sia praticamente impossibile condurre in porto una ricerca accurata su argomenti politici, sociali, giuridici ed economici senza inciampare nel problema dell'ideologia. Spesso la questione viene accantonata con la sbrigativa osservazione «Io non adotto un approccio ideologico». Questa pretesa è assurda. Max Weber afferma infatti: «Lasciar parlare i fatti è il metodo più sleale» (Weber 1966: 29). Le relazioni umane non producono solo fatti, ma anche interpretazioni di fatti, circostanza della quale gli storici più accurati sono consapevoli. Edward Carr osserva: «Lo storico non è né l'umile schiavo né il tirannico padrone dei fatti. Il rapporto tra lo storico e i fatti si svolge su un piano di parità, di scambio reciproco. ... lo storico è perpetuamente intento ad adeguare i fatti all'interpretazione e l'interpretazione ai fatti. È impossibile assegnare un primato all'uno o all'altro momento» (Carr 1966: 35). La medesima considerazione vale naturalmente anche per il sociologo, l'economista, il giurista, il politologo e l'antropologo.

Aggiungo una breve osservazione sull'attualità di questo dibattito. Sin dalla seconda metà del secolo scorso si è manifestato un consenso crescente alla tesi della «fine delle ideologie», così che persino chi fa politica attiva a volte afferma, a sproposito, che le sue proposte non 
hanno nulla a che vedere con l'ideologia. Questa ondata conformista ha avuto come effetto, sebbene non ne sia la causa principale, di ridurre la portata e i contenuti dei programmi dei partiti democratici: l'orizzonte è quello del prossimo governo, la conquista del potere nazionale. Ci si aggrappa al pragmatismo per giustificare proposte di breve respiro, dimenticando le questioni maggiori, come la crisi dell'ordine internazionale, il riarmo atomico e la crisi ambientale, alcune incombenti minacce sul futuro della specie umana. Eppure, la crisi dell'ordine internazionale dopo la fine della guerra fredda avrebbe dovuto provocare un brusco risveglio per i sonnambuli della politica. Prima la disgregazione dell'URSS, poi il declino dell'egemonia americana e l'affermazione di nuove potenze globali, come la Cina e l'India, hanno mostrato che al vecchio ordine egemonico bipolare si sta sostituendo un'accesa competizione tra grandi e piccole potenze che giustificano sempre più la loro politica estera e interna con il nazionalismo. Perché l'ideologia nazionale ritorna a dominare la politica internazionale, mentre le altre ideologie stanno battendo in ritirata? Occorre trovare una risposta a questo interrogativo.

Esaminiamo in primo luogo alcune basi teoriche. Affrontiamo la questione prendendo in considerazione un illuminante saggio del 1976, di Georg von Wright, un filosofo finlandese che ha continuato, a Cambridge, a sviluppare l'approccio analitico alla filosofia iniziato da Wittgenstein. In un saggio dal titolo «Il determinismo e lo studio dell'uomo», von Wright si propone di studiare la logica dell'azione umana intenzionale, un problema che è di scarso interesse per le scienze della natura, come la fisica, il darwinismo e la biologia. «La scienza dell'uomo - afferma von Wright - è in primo luogo uno studio di fenomeni che cadono sotto il 'dominio' di istituzioni e regole sociali» (von Wright: 1976: 234). Lo studio della complessa realtà sociale diventa possibile se ci si concentra sul comportamento intenzionale di un singolo individuo. Per questo von Wright propone un sillogismo pratico, che illustra nel modo seguente: $\ll \mathrm{A}$ intende $p$ (per esempio, andare a teatro domani); $\mathrm{A}$ ritiene di non poter fare $p$ se non fa $q$ (per esempio, prenotare un biglietto in anticipo). Quindi A si dispone a fare q» (p. 236). Non è necessario approfondire questa analisi, che riguarda la decisione in vista di un'azione che si realizzerà in futuro, dunque nel corso di un processo temporale, la storia, come avviene anche per il pensiero ideologico. Ricordiamo solo che nel corso di questo saggio von Wright osserva che le leggi sociali sono per definizione soggette al cambiamento storico, 
pertanto «le 'leggi' sociali non sono generalizzazioni dall'esperienza, ma schemi concettuali per l'interpretazione di situazioni storiche concrete. ... si può dire che la ricerca sociale occupa una posizione intermedia fra filosofia e storia. Essa può muoversi in direzione dell'uno o dell'altro dei due poli, ma non può vivere un'esistenza autonoma separata da uno dei due» (p. 260). Si tratta di un'osservazione importante, che suggerisce la necessità di una sempre più stretta collaborazione tra le diverse discipline. A queste oggi occorre aggiungere gli sviluppi della scienza e della tecnologia, che si stanno dimostrando, in particolare l'informatica e la biologia, come determinanti cruciali dei comportamenti sociali, data l'accelerazione crescente del progresso tecnologico e le sue ripercussioni sulla vita quotidiana. La paleo-archeologia, negli studi sull'origine dell'umanità, ha compiuto strabilianti scoperte grazie alla datazione con il metodo del radiocarbonio dei fossili e lo studio del DNA dei resti umani.

Recentemente un altro filosofo analitico, John Searle, ha sviluppato un importante metodo per lo studio del comportamento intenzionale in una serie di convincenti analisi (Searle 2006 e 2010). Searle estende lo studio del comportamento umano intenzionale dall'individuo alla collettività, mediante un netto rifiuto dell'individualismo metodologico che sino a quel momento era adottato da quasi tutta la comunità degli scienziati sociali. «Nell'intenzionalità collettiva, afferma Searle (2010: 57), non si può richiedere che ogni intenzionalità individuale sappia qual è l'intenzionalità degli altri partecipanti. In forme complesse di gioco di squadra o comportamento cooperativo, solitamente non si sa nei dettagli cosa stanno facendo gli altri. Tutti devono credere che stanno condividendo un obiettivo collettivo e intendono fare la loro parte nel raggiungere quell'obiettivo». Possiamo proporre, come esempio, il comportamento dei giocatori di una squadra di calcio. Ogni giocatore svolge un certo ruolo nella consapevolezza che la sua prestazione si inserisce in uno schema di comportamenti collettivi rivolti a uno scopo preciso: segnare un goal agli avversari. Un altro esempio è la divisione del lavoro in fabbrica, dove ogni operaio svolge una mansione specifica, non necessariamente la stessa dei suoi compagni di lavoro, ma è rivolta all'ottenimento di un certo prodotto. Al contrario, il comportamento degli imprenditori che orientano la loro azione all'ottenimento del massimo profitto non può essere considerato un comportamento collettivo, sebbene il loro obiettivo sia il medesimo: sono le regole del mercato a coordinare la loro azione, ma non è necessario che ogni imprenditore si 
coordini con gli altri. «Per potere partecipare a un comportamento collettivo - osserva Searle (2010: 68) - io devo credere (o assumere o presupporre) che gli altri stiano cooperando con me». In breve, affinché esista una intenzionalità collettiva devono esistere uno scopo, una meta o un ideale comune da raggiungere.

Consideriamo ora molto succintamente un secondo insieme di nozioni elaborate da Searle che saranno particolarmente utili alla nostra indagine. Mi concentro sul rapporto tra intenzionalità collettiva e istituzioni. L'importanza di questa relazione è indicata dal sottotitolo che Searle ha proposto per il suo libro: «La struttura della civiltà umana». In breve, le istituzioni sono il «cemento» che tiene unita l'intera società umana nel corso del suo rinnovamento. Alla base di quest'affermazione, Searle pone giustamente il linguaggio, come l'istituzione fondamentale necessaria per la creazione di tutto il complesso sistema di interdipendenze in cui viviamo. «Si può avere una società che ha un linguaggio, ma non uno stato, la proprietà privata o il denaro. Di contro, però, non si può avere una società che ha uno stato, la proprietà privata e il denaro ma non ha il linguaggio» (2010: 81). La peculiare natura del linguaggio consente agli umani di creare istituzioni che li distinguono da tutte le altre specie viventi. Nel paleolitico, homo sapiens può rappresentare nella sua mente la realtà esterna (ad esempio osservare che esiste un fiume che ostacola il suo cammino) e può concepire un'azione collettiva con i suoi compagni di avventura (come costruire una zattera) per attraversare il fiume. «Con l'aggiunta di una sintassi linguistica all'intenzionalità animale consentiamo ai parlanti di fare qualcosa che gli animali non linguistici non sono in grado di fare» (2010: 104). Possiamo ora proporre un semplice esempio di come può nascere un'istituzione umana sulla base della struttura logica con la quale il linguaggio la crea. Immaginiamo un matrimonio tra due persone di fronte ad un ufficiale dello stato civile o religioso. Una volta celebrato il matrimonio con le formule di rito si avranno anche conseguenze giuridiche, diritti e doveri dei due coniugi. Searle indica la formula logica dei nuovi impegni sociali derivanti dal rito del matrimonio e di altre istituzioni: «Le regole costitutive hanno la forma ' $\mathrm{X}$ ha valore di (counts as) $\mathrm{Y}$ in C'. Per esempio, proferire una certa enunciazione $\mathrm{X}$ in questo contesto C ha valore di (counts as) fare la promessa Y» (2010: 106; corsivi originali). Un ulteriore esempio è il seguente: una banconota da 100 euro $(\mathrm{X})$ ha un potere d'acquisto (counts as) pari a un certo paniere di beni $(\mathrm{Y})$ nell'Unione monetaria europea $(\mathrm{C})$. Gli esseri umani sono così in 
grado di modificare la realtà sociale in cui vivono creando istituzioni che consentono di regolare rapporti sociali complessi, come il mercato, le chiese, le università, lo stato e le relazioni tra stati. Le istituzioni rappresentano una forma di potere, il potere deontico. «Con le convenzioni del linguaggio, abbiamo già una deontologia; abbiamo già gli impegni nel senso pubblico vero e proprio che combina l'irrevocabilità e l'obbligo. Il linguaggio è la forma fondamentale della deontologia pubblica» (2010: 108). In conclusione: «tutta la realtà istituzionale è creata da dichiarazioni ... Il linguaggio, però, non è creato da una dichiarazione» (2010: 145).

Una volta che abbiamo chiarito i rapporti tra intenzionalità individuale, intenzionalità collettiva e creazione delle istituzioni sociali, il cemento della civiltà, possiamo discutere più agevolmente del ruolo delle ideologie nella società e nelle scienze sociali. Ricordiamoci del 'sillogismo pratico': esso descrive una relazione tra mezzi e fini: se voglio andare a teatro domani, devo comprare un biglietto d'ingresso. Se ora consideriamo le grandi ideologie formatesi nell'età moderna, vale a dire il liberalismo, la democrazia, il socialismo e il nazionalismo, possiamo indicare, rinunciando a una descrizione minuziosa dei loro contenuti, la relazione tra il valore essenziale, o primario, che caratterizza ogni ideologia politica moderna e i mezzi istituzionali con i quali i loro propugnatori pensavano o pensano di realizzare il valore che difendono primariamente. Ho indicato solo quattro ideologie per semplificare, forse eccessivamente, la discussione. Tuttavia, non è difficile applicare questi concetti ad una serie ampia di partiti politici che difendono, sotto diverse bandiere, programmi misti, come ad esempio i partiti cristiano-sociali, i partiti liberal-socialisti, i partiti liberal-conservatori, i partiti nazional-socialisti, ecc. Le quattro ideologie che ho elencato le raggruppo per semplicità sotto l'etichetta 'ideologie tradizionali'. Mi sembra che meritino questa definizione perché hanno caratterizzato la storia moderna con le prime manifestazioni del liberalismo inglese e la Rivoluzione gloriosa e, in seguito, con le ideologie affermatesi prepotentemente durante la Rivoluzione francese e la rivoluzione industriale.

Lo sviluppo del liberalismo è noto. Viene spesso fatto risalire alle rivendicazioni dei diritti di alcuni nobili e della parte più ricca della nascente borghesia nei confronti del sovrano di diritto divino. Si tratta di una fase che viene definita dei diritti negativi, cioè la rivendicazione delle libertà individuali dal potere coercitivo dello stato. A questa fase è seguita quella della libertà positiva, perché, come afferma Guido De 
Ruggero, la libertà non è arbitrio «ma capacità dell'uomo di determinarsi da sé ... ogni dipendenza naturale o coattiva è negata e subentra al suo posto quella che la coscienza dei doveri verso sé e verso gli altri pone spontaneamente» (De Ruggero 1962: 339). Cambia così anche la natura dello stato. «Lo stato, organo della coazione per eccellenza, è divenuto la massima espressione della libertà; il nemico tradizionale degli individui s'è modellato nella forma della coscienza individuale » (De Ruggero 1962: 341). In questo processo di trasformazione dello stato, mediante il riconoscimento di una libertà positiva a fianco di quella negativa, si perfeziona anche la simbiosi tra liberalismo e democrazia (il contesto $\mathrm{C}$ di Searle). La più evidente e precisa manifestazione di questo felice e inevitabile connubio è probabilmente rappresentato da John Stuart Mill, e dai suoi due saggi, il primo On liberty, pubblicato nel 1859, e il secondo Representative Government, pubblicato nel 1861. Nel linguaggio politico contemporaneo è ormai consuetudine discutere della liberal-democrazia come una forma di governo che garantisce ai cittadini una serie di diritti fondamentali e la loro piena partecipazione - senza distinzioni di classe, ricchezza, sesso, cultura, etnia o religione - alla formazione del governo, che solo con il consenso esplicito dei cittadini può considerarsi rappresentativo.

Per quanto riguarda il socialismo, se prendiamo in considerazione le sue prime espressioni rappresentate dal pensiero di Saint-Simon, Fourier, Owen, è possibile indicare il suo valore primario nella giustizia sociale, generata dalla critica dello sfruttamento dei lavoratori nelle fabbriche e dalle condizioni insalubri degli agglomerati urbani in cui erano costretti ad ammassarsi. Tuttavia, nel Manifesto dei comunisti, del 1848, Marx e Engels denunciano il pensiero dei primi socialisti come utopistico, e indicano il comunismo come alternativa. Fondano la loro analisi sulla divisione in classi della società e la formazione di un mercato capitalistico mondiale come base materiale della vita associata (il contesto C) e propongono, come alternativa alla organizzazione capitalistica della produzione, l'abolizione della proprietà privata dei mezzi di produzione mediante una rivoluzione violenta che ponga fine al dominio del potere della borghesia. Lo stato, per Marx e Engels, non è altro che il comitato d'affari della borghesia. Gli sviluppi successivi, nella seconda parte del secolo XIX e i primi decenni del secolo XX, sono noti. Una parte del movimento operaio ha scelto la via parlamentare e democratica per accrescere e consolidare il potere dei lavoratori nello stato, come è avvenuto con la socialdemocrazia tedesca. Un'altra parte del 
movimento operaio ha scelto la via indicata da Lenin per la conquista del potere nella Russia zarista e la successiva creazione dell'URSS, la patria dei lavoratori.

Non è necessario indicare con più precisione gli sviluppi del liberalismo, della democrazia e del socialismo. Per i fini che mi propongo - e che indirettamente riguardano anche gli sviluppi extra-europei delle quattro ideologie - è sufficiente una breve indicazione del rapporto tra i valori primari di queste ideologie e i mezzi che i partiti e i governi hanno concepito e realizzato per conseguirli. Il solo problema che resta in sospeso, e che va affrontato, è il rapporto tra le tre ideologie tradizionali e il nazionalismo. Il nazionalismo ha uno sviluppo apparentemente simile alle tre ideologie precedenti, ma il suo rapporto con i loro valori è complesso e opaco. Se prendiamo in considerazione la fase embrionale del nazionalismo, i movimenti di popolo che hanno portato all'unificazione italiana e tedesca, troviamo una forma di pensiero che riesce a fare convivere nazionalismo e cosmopolitismo. Mazzini ha fondato quasi contemporaneamente la 'Giovine Italia' e la 'Giovine Europa' nella convinzione che, una volta liberata l'Italia dall'oppressione dell'Impero austriaco e scelta la forma repubblicana di governo, si sarebbe automaticamente risolto il problema della convivenza pacifica tra le nazioni europee. Tra repubbliche, con un governo eletto dal popolo, non vi è ragione alcuna per temere delle guerre. Non diversamente da Mazzini pensano i democratici a lui contemporanei e ancora oggi è molto diffusa l'ideologia liberal-democratica dei rapporti internazionali (si pensi alla 'Fine della storia' di Fukuyama: è il trionfo della liberal-democrazia internazionale dopo la fine della guerra fredda). In Germania Friedrich List, negli stessi anni in cui Mazzini iniziava la sua attività rivoluzionaria, proponeva l'unificazione del mercato tedesco, poi realizzata con lo Zollverein, come primo passo verso l'unificazione politica e l'industrializzazione della Germania. L'area tedesca era infatti costretta a subire il dominio economico inglese, la cui manifattura esportava prodotti a un prezzo talmente basso da impedire la formazione di un'industria nazionale. Il teorico dell'industria nascente proponeva come soluzione un protezionismo momentaneo, sino a che anche l'industria tedesca sarebbe stata competitiva nel mercato mondiale. $\mathrm{Al}$ termine del processo, secondo List, si sarebbe formato un grande mercato cosmopolitico di nazioni libere e pacifiche.

La seconda parte dello sviluppo del nazionalismo è nota. Una volta che la rivoluzione industriale si estese dalla Gran Bretagna a tutte 
le altre nazioni europee e agli Stati Uniti, è iniziata un'accanita corsa alla conquista delle colonie che ha indotto i partiti tradizionali - liberali, democratici e socialisti - a diventare complici della politica di potenza nazionale. I leader di questi partiti non hanno compreso la natura di un processo che stava conducendo i popoli europei verso la catastrofe (sui rapporti tra liberalismo e socialismo con l'imperialismo, Montani 2019a). I liberali e i socialisti non hanno saputo reagire ai primi sintomi di una politica che si è presto trasformata in uno scontro tra grandi potenze sui campi di battaglia della prima guerra mondiale. Gli stati nazionali europei avevano consentito di integrare al loro interno tutte le categorie sociali esistenti, grande borghesia, piccola borghesia, lavoratori dell'impresa e di altri settori pubblici e privati. Era stato possibile un primo compromesso tra liberalismo, democrazia e socialismo. Ora il popolo era veramente unito nello stato ma questo stato, che si proclamava sovrano, concepiva i rapporti internazionali come conflittuali, dove solo la forza militare poteva consentire di difendere l'indipendenza nazionale (il valore primario del nazionalismo). Per affermare il suo primato, lo stato nazionale chiedeva ai propri cittadini di morire per la difesa della patria. La storia di questi avvenimenti è ricca di testimonianze di uomini uniti da valori culturali, religiosi e politici comuni, come testimoniano gli anni della belle époque, con un'intensa fioritura di espressioni artistiche comuni a tutti i paesi europei, lo sviluppo straordinario delle scienze naturali, sociali e della filosofia, la creazione delle prime forme di organizzazioni sovranazionali, come le internazionali operaie. Dopo pochi anni, sono gli stessi uomini che hanno condiviso una lunga epoca di pace a combattere su fronti opposti. Right or wrong, my country. Il nazionalismo è una forma moderna di religione alla quale si innalzano altari e si sacrificano vittime umane. Il nazionalismo si è impadronito del sentimento del sacro che alberga in ogni essere umano, per trasformarlo in uno strumento di potere.

Gli anni Venti e Trenta del secolo scorso sono da molti studiosi, ad esempio Carr (1981), considerati come una tragica sequenza di crisi politiche, economiche e sociali che sono sfociate nella seconda guerra mondiale, una seconda immensa catastrofe, della quale le immagini delle macerie delle città distrutte, delle bombe atomiche cadute su Hiroshima e Nagasaki, di Auschwitz e degli altri campi di sterminio restano incancellabili nella memoria non solo di chi ha vissuto quella tragedia, ma anche delle successive generazioni. Com'è potuto avvenire tutto questo? Come è potuto avvenire che l'eredità spirituale dell'uma- 
nesimo, del rinascimento e dell'illuminismo e l'idea del progresso dell'umanità abbiano prodotto 'il male assoluto'?

È necessario cercare una risposta a questi interrogativi e, in questa prospettiva, può essere utile approfondire ulteriormente la conoscenza del pensiero ideologico. Dai pochi cenni fatti in precedenza sulla struttura delle ideologie tradizionali, si può intuire come il rapporto tra i fini dell'azione collettiva intenzionale - i valori della libertà, dell'eguaglianza politica, della giustizia sociale e dell'indipendenza nazionale - e i mezzi non vi sia una relazione univoca, ma un largo margine di interpretazione e di incertezza (il contesto C). Il compito di un partito o di un uomo di stato è guidare una comunità verso un futuro incerto, perché il cammino che l'umanità intende percorrere non può essere dedotto da un preciso modello, da una conoscenza razionale, simile a quanto fanno gli scienziati della natura. Come ha osservato von Wright, le scienze sociali non possono fare a meno di considerarsi come un campo della conoscenza intermedio rispetto ad altre discipline, come la storia e la filosofia (a cui occorre aggiungere le scienze della natura e la tecnologia). Per questo, lo scienziato sociale deve riconoscere i limiti del suo impegno professionale. Max Weber, giustamente, introduce una netta distinzione tra politica attiva e studio del comportamento umano. «La politica non si addice all'aula d'insegnamento ... la cattedra non è per i profeti e i demagoghi» (Weber 1966: 28-9). Politica attiva e studio del comportamento politico sono due professioni distinte. Possono coincidere eccezionalmente, nelle fasi embrionali di un pensiero politico, come è avvenuto con Thomas More, Locke, Mazzini, List, Marx e Lenin.

Il primo contributo importante alla comprensione del pensiero ideologico è la scoperta, fatta da Marx, del fenomeno della falsa coscienza. La sua critica al pensiero liberale e in particolare al capitalismo è nota e non è qui il caso di ricordarla. Tuttavia, mi sembra che sia possibile affermare che tutte le ideologie corrono questo rischio e il marxismo non è certamente sfuggito al pericolo della falsa coscienza, come dimostra la storia dell'URSS specialmente nella fase della dominazione staliniana, quando la verità propagata dal regime era difesa dalle critiche mediante l'imprigionamento, la tortura e l'uccisione dei 'nemici del popolo'. Il pericolo della falsa coscienza è un fenomeno permanente del pensiero politico, che può essere attenuato, ma non eliminato del tutto, sia con il contributo delle scienze sociali sia con la rinuncia della politica all'uso della violenza fisica e all'intimidazione degli avversari. Ė pertanto il rapporto tra politica e violenza il problema che 
va indagato più a fondo, perché, nonostante i progressi compiuti in ogni campo del sapere e della vita civile, la violenza resta ancora oggi un'oscura protagonista della vita collettiva, sia tra individui (persino nella famiglia) sia tra comunità umane (neri e bianchi, maschi e femmine, credenti e laici, etnia A e etnia B, ecc.), sia tra stati. La filosofia, con l'aiuto delle scienze sociali, si sta occupando sempre più del poliedrico problema della violenza (Magnani 2012).

A mio avviso, è la politica che potrà dare un contributo decisivo alla creazione di una società dove i rapporti individuali siano sempre più liberi dal dominio di pulsioni violente e da un potere politico che le alimenta. Per esaminare questo problema, mi sembra utile accennare ai recenti sviluppi della politica internazionale dopo la fine della guerra fredda. Il secondo dopoguerra è stato caratterizzato da un sistema mondiale dominato dalle due superpotenze, USA e URRS, che nel bene e nel male hanno assicurato una lunga epoca di stabilità internazionale, sebbene non senza tensioni tra di loro e nelle rispettive aree d'influenza, dove a volte il dissenso è stato represso con la forza. Tuttavia, l'equilibrio del terrore $(\mathrm{MAD})$ ha impedito che il sistema bipolare di governo del mondo degenerasse in guerra aperta. La caduta del muro di Berlino ha aperto un primo spiraglio al ritorno del nazionalismo, sia nei paesi della ex-URSS sia nella ex-Jugoslavia, dove un'Unione europea attonita e impaurita ha visto ritornare ai suoi confini i campi di sterminio e la pulizia etnica. Nei primi decenni del secolo XXI è poi apparso evidente che gli USA non erano più in grado di svolgere la vecchia funzione di 'stabilizzatore' dell'ordine internazionale. Nuove grandi potenze stanno occupando la scena mondiale, come la Cina, l'India, il Brasile, e la stessa Russia, determinata a recuperare il ruolo da protagonista del passato. In breve, la nuova politica internazionale si sta avviando verso un sistema multipolare, dominato da grandi potenze che si propongono di affermare un proprio primato: a 'America First' si accompagna 'China First', 'Russia First', ecc. Inoltre, come la storia dello scorso secolo insegna, la politica di potenza si deve necessariamente fondare su una forte coesione interna e sulla forza militare esterna. Negli USA ritorna una certa simpatia per il suprematismo bianco, in Cina si favorisce l'omogeneizzazione etnica Han, e si perseguitano gli Uiguri; in India si favorisce il dominio dell'etnia Hindu sulla popolazione musulmana e in Africa e nel Medio Oriente si manifestano continuamente contrasti etnici e religiosi che degenerano facilmente in genocidi. Se a questo scenario, aggiungiamo la corsa verso nuovi armamenti atomici, annunciata da 
Putin e Trump, possiamo costatare con preoccupazione che si sta mettendo in moto una crescente tensione internazionale simile a quella che ha condotto alla prima e alla seconda guerra mondiale. Il fatto che il nazionalismo di seconda generazione sia ancora nella sua fase embrionale non significa che sia meno pericoloso di quello che l'ha preceduto. È una malattia mortale che va contrastata prima che sia troppo tardi.

Non intendo qui inoltrarmi in una discussione approfondita delle cause della crisi dell'ordine internazionale e delle spiegazioni che stanno emergendo, che sono sostanzialmente basate sulla concezione tradizionale delle relazioni internazionali: alcuni auspicano il ritorno a un ordine liberal-democratico che ripristini il multilateralismo, mentre i sostenitori del pensiero marxista e socialista mirano a un ordine internazionale più giusto mediante un maggior controllo - nazionale - della finanza e delle imprese multinazionali. Non voglio qui approfondire questa discussione; non è necessario (per chi fosse interessato, rimando a: Montani 2019b). Mi concentrerò sulla linea argomentativa adottata in questa conversazione riguardante il ruolo delle ideologie nel pensiero politico, nella convinzione, o nella speranza, di poter indicare una linea d'azione efficace. Cercherò di dimostrare che il dibattito politico internazionale deve prendere in considerazione il problema del futuro dell'umanità, perché non si può nascondere impunemente ai cittadini che le grandi potenze stanno avviando una nuova fase di corsa agli armamenti nucleari, con sistemi di controllo pericolosissimi, basati sull'intelligenza artificiale; a questa minaccia si aggiunge quella del cambiamento climatico e della sesta estinzione delle specie viventi, tra le quali va naturalmente inclusa la specie umana. L'orizzonte del dibattito si deve dunque spingere oltre i limiti imposti da una politica basata sull'ideologia nazionale e su un ordine internazionale aperto al confronto militare, economico e tecnologico tra grandi e piccole potenze. È necessario prendere in considerazione la prospettiva cosmopolitica come alternativa al nazionalismo di seconda generazione.

Non propongo un progetto utopistico, nel senso di una fantasia irrealizzabile. Mi considero un realista e mi muovo, per quanto possibile, sul terreno del realismo politico che risale a Machiavelli. Pertanto, prenderò ora in esame due ideologie nuove, rispetto alle quattro che ho definito 'ideologie tradizionali'. Sono ideologie che sono sorte e si sono affermate dopo la seconda guerra mondiale, la prima come risposta alla grande catastrofe e la seconda come risposta ai guasti crescenti provocati dall'industrialismo sulle risorse naturali del Pianeta: in breve, il 
federalismo sovranazionale europeo e l'ecologismo o ambientalismo.

L'unificazione politica dell'Europa è stata concepita come necessaria già nel corso della seconda guerra mondiale da due pionieri, Jean Monnet e Altiero Spinelli, uno degli autori del Manifesto di Ventotene (1941). Tuttavia, mentre Jean Monnet ha svolto un ruolo cruciale nella creazione della prima comunità politica sovranazionale, la CECA (Comunità europea del carbone e dell'acciaio), Spinelli ha svolto un'azione altrettanto cruciale per trasformare la Comunità europea in federazione. Mentre l'azione di Monnet ha concentrato l'attenzione dei politici e dei governi sulla strategia delle riforme parziali, o dei piccoli passi, oggi sostenuta da tutti gli ambienti europeistici, Spinelli ha fondato la sua strategia sul superamento della sovranità assoluta degli stati europei, mediante il metodo costituente. Ricordo l'affermazione cruciale del Manifesto di Ventotene: «La linea di divisione fra partiti progressisti e partiti reazionari cade ormai non lungo la linea formale della maggiore o minore democrazia, del maggiore o minore socialismo da istituire, ma lungo la sostanziale nuovissima linea che separa quelli che concepiscono come fine essenziale della lotta quello antico, cioè la conquista del potere politico nazionale - e che faranno, sia pure involontariamente, il gioco delle forze reazionarie lasciando solidificare la lava incandescente delle passioni popolari nel vecchio stampo, e risorgere le vecchie assurdità - e quelli che vedranno come compito centrale la creazione di un solido stato internazionale, che indirizzeranno verso questo scopo le forze popolari e, anche conquistato il potere nazionale, lo adopereranno in primissima linea come strumento per realizzare l'unità internazionale». La linea di divisione del Manifesto di Ventotene non è sempre stata seguita con coerenza dai federalisti europei nei vari paesi del continente, ma è certamente stata la fonte primaria di ispirazione dei federalisti italiani, che hanno comunque avuto un ruolo centrale nell'evoluzione della strategia federalista nell'Unione europea (sul pensiero dei federalisti italiani cfr. Cultura della pace e cultura della guerra di Mario Albertini, 1999: 151-74). Ė vero che oggi l'Unione europea è ancora una federazione incompiuta, ma non sembra contestabile il fatto che alcune sue istituzioni, come la Commissione europea, la Corte di giustizia, il Parlamento europeo e la Banca centrale europea siano istituzioni federali, o sovranazionali. Per quanto imperfetta, l'Unione europea è la sola istituzione sovranazionale esistente e, in una situazione di grave crisi della politica internazionale, può svolgere un ruolo guida verso un nuovo ordine internazionale pacifico. 
La seconda corrente di pensiero che rifiuta, non sempre coerentemente, il nazionalismo è l'ambientalismo o ecologismo. I primi segnali della formazione di un pensiero ecologista risalgono agli anni Sessanta nei paesi anglo-sassoni. Tuttavia, il movimento ecologista si è via via ingrossato ed ha accresciuto il suo potere politico nelle istituzioni nazionali di alcuni paesi europei importanti, come la Germania e la Francia, oltre che negli USA nelle file del partito democratico. La situazione è mutata in tempi più recenti, quando, grazie alle frequenti conferenze dell'ONU sul surriscaldamento climatico, agli angosciosi appelli degli scienziati e alla rivolta dei giovani, finalmente la questione ambientale si è imposta all'attenzione della politica mondiale. L'ideologia dell'ambientalismo ha avuto molti precursori e teorici. Mi limito a citare due analisi rilevanti. La prima è di Hans Jonas (1993), Il principio responsabilità, pubblicato nel 1979, dove il rapporto tra natura umana, tecnologia e ambiente viene esaminato sotto l'aspetto etico e si propone 'il principio responsabilità', dunque l'impegno che ogni individuo e ogni generazione devono assumere per rendere possibile la vita sul Pianeta (ecco il valore primario dell'ecologismo) alle generazioni che verranno. E anche su questa base che successivamente è stato formulato il concetto di 'sviluppo sostenibile', oggi adottato da quasi tutti gli studiosi, sia delle scienze sociali che delle scienze naturali, come principio guida delle strategie ambientali. L'ecologismo ha fruito, come piattaforma organizzativa, delle conferenze dell'ONU. Oggi, i Sustainable Development Goals sono diventati un punto di riferimento per tutti i governi e i politici che intendono affrontare seriamente la questione ambientale. Il secondo autore che ha contribuito a sviluppare un'etica e un'ideologia ambientale è Stephen Gardiner. La sua analisi chiarisce efficacemente come i governi nazionali riescano a scaricare sulle generazioni future i costi del risanamento ambientale che dovrebbe essere realizzato subito per ridurre i costi che, se elusi, graveranno sulle generazioni future. Cito solo un passo delle sue conclusioni: «In modo preminente, il cambiamento climatico è uno dei numerosi problemi globali contemporanei che gettano dubbi sulla tradizionale strategia filosofica della costruzione della giustizia sociale sulla base di un solo autosufficiente stato nazionale. Se siamo veramente entrati in una nuova epoca sulla terra, un'era geologica dominata dall'umanità - l'antropocene - allora questo modello sembra seriamente insufficiente e forse invecchiato senza rimedio. I teorici dovrebbero chiedersi se ciò richiede una revisione delle loro grandi visioni di etica e giustizia» (Gardiner 2011: 435). 
Possiamo ora compiere l'ultimo passo verso la fine di questo lungo intervento. È utile ridiscutere delle varie ideologie, i cui valori primari - un tentativo di dire l'indicibile, secondo von Wright - si manifestano nel corso della storia e, come suggerisce il sillogismo pratico, possono convivere, non senza contrasti e lotte tra i loro sostenitori, per promuovere le politiche e le istituzioni che sono necessarie per tradurli in realtà. Ora prenderemo in considerazione il rapporto tra ideologia e utopia, come ha fatto Karl Mannheim (1957), il primo studioso moderno di questo fenomeno, ma come non hanno fatto i politologi, i cui studi sono dedicati preferibilmente al solo pensiero ideologico. La ragione per la quale è utile discutere del rapporto tra i due concetti è difesa molto efficacemente dal filosofo Paul Ricœur, che ricorda come si sia troppo frettolosamente annunciata «la morte delle ideologie e rinunciato così a ogni progetto di società. $\mathrm{Ma}$ certe idee sostenute da queste utopie di un avvenire migliore, quella della 'pace perpetua' per esempio, nel senso di Kant o di Fichte, non devono forse essere salvate? Non è sufficiente dire, come Jean-François Lyotard, che i 'grands récits' di emancipazione sono finiti, o come Fukuyama che 'la storia è finita'. Non si tratta solo che queste sono prospettive della disperazione; che ne è della dimensione del progetto senza la quale non si può avere alcuna azione politica?”》 (Ricœur 2017: 116-7).

Se prendiamo in considerazione il progetto a lungo termine delle due ideologie moderne, appena ricordate, si può intravedere un approdo cosmopolitico per entrambe. Per quanto riguarda la 'linea di divisione tra progresso e reazione', tracciata nel Manifesto di Ventotene, il traguardo conclusivo non è solo quello degli Stati Uniti d'Europa, ma 'un solido stato internazionale' che non è necessariamente europeo. In effetti, lo stesso Manifesto di Ventotene accenna brevemente anche alla creazione, in un tempo lontano, della federazione mondiale, dunque della prospettiva kantiana della 'pace perpetua'. Oggi l'ONU, con tutte le sue pecche, in particolare quella di fondare i rapporti internazionali sulla sovranità assoluta degli stati nazionali, dunque sugli armamenti e sulla guerra, ha approvato nel 1948 la Dichiarazione universale dei diritti dell'nomo, una serie di enunciazioni formali la cui realizzazione è stata affidata ai governi nazionali, ma non a una Corte sovranazionale, a cui i cittadini del mondo possano rivolgersi per chiedere giustizia nei confronti di un potere oppressivo. In quale direzione occorre riformare l'ONU? Si potrebbe pensare che l'Unione europea, che a sua volta ha approvato una Carta dei diritti fondamentali dell'Unione europea e che 
ha istituito la 'cittadinanza europea' che attribuisce a ogni cittadino il diritto di studiare, lavorare e circolare in tutti i paesi dell'Unione, si faccia promotrice del riconoscimento di un simile diritto per ogni abitante del Pianeta. Molti giovani, oggi, già pensano e agiscono come cittadini del mondo. L'UE potrebbe diventare il propulsore per una riforma cosmopolitica del diritto di cittadinanza, per il riconoscimento dello status giuridico di cittadino del mondo.

Un analogo orizzonte cosmopolitico si intravvede nel pensiero ecologista. Non avrebbe nessun senso una lotta politica per salvaguardare la purezza della natura in un solo paese. L'inquinamento dell'aria e dell'acqua non ha confini, come non hanno confini i moderni canali di comunicazione, le infezioni e lo sterminio delle specie animali e vegetali. Non possiamo rassegnarci alla fine della vita sul Pianeta. Gli stati sono sorti per difendere la vita, per garantire la sicurezza dei loro cittadini nei confronti dei pericoli provenienti dall'interno e dall'esterno. Tuttavia, oggi gli stati nazionali non sono più in grado di svolgere questo compito primario. Ha ragione Edgar Morin quando afferma: «Non esiste civiltà che non abbia un fondo di barbarie. Essendo la barbarie un ingrediente della civiltà, possiamo solo resisterle, non sopprimerla. Gli esseri rivelano il proprio meglio o peggio nei periodi di crisi, di conflitto, di disastro» (Morin 2018: 93). Dobbiamo reagire alla crisi, alla ignavia dei governi nazionali. Molti intellettuali e politici «ignorano che la comunità di destino di tutti gli umani sulla Terra-Patria esige una coscienza comune di Terra-Patria che invilupperebbe le patrie senza sopprimerle» (p. 144). Per contenere i residui di barbarie che albergano nella natura umana, la Terra-Patria dovrà essere abitata da cittadini del mondo.

Possiamo ora ripetere il medesimo esercizio per le quattro ideologie tradizionali di cui abbiamo discusso in precedenza. Per la liberaldemocrazia si potrebbe affermare che la sua utopia, l'obiettivo di lungo periodo, sia uno stato mondiale di diritto regolato da una 'governance' democratica. L'ONU ha approvato, il 19 settembre 2012, una Dichiarazione sulla Rule of Law at the National and International Levels. La Dichiarazione è apprezzabile per i princìpi enunciati, tuttavia, come avviene per altre Dichiarazioni dell'ONU, si lascia ai governi nazionali la responsabilità finale della loro adozione. Nessun "potere' sovranazionale è previsto, così che la violazione eventuale dello stato di diritto da parte di un governo nazionale non potrà essere sanzionata. Per quanto riguarda il socialismo e il comunismo, la discussione è più 
complessa. Per fare un esempio, Jonas afferma che l'utopia marxista consiste nella società senza classi. Tuttavia, dopo il fallimento dell'URSS e dell'esperimento della abolizione della proprietà privata dei mezzi di produzione, ogni proposta di società che realizzi la giustizia sociale deve fare i conti con la storia. La Cina ha un governo che si dichiara comunista, ma ha introdotto il sistema economico capitalista come veicolo del suo formidabile sviluppo economico. Forse, ai nostri fini, è sufficiente indicare una società cosmopolitica che, all'interno di uno stato che rispetti i principi della liberal-democrazia, riduca le diseguaglianze tra le ricchezze dei cittadini al minimo, promuovendo efficaci politiche per la lotta alla povertà e la riduzione del tempo di lavoro.

Resta da discutere il caso dell'ideologia nazionale. Come abbiamo visto anche questa ideologia ha avuto con Mazzini e List una fase utopica, cioè l'ipotesi che fosse possibile un mondo popolato da nazioni pacifiche, aperte al libero scambio internazionale. Quest'utopia si è tuttavia scontrata con la dura realtà della politica di potenza e la degenerazione dell'ideale nazionale in odio tra popoli, che ha raggiunto il suo apice con la prima e la seconda guerra mondiale. Oggi, se si vuole indicare il punto di arrivo di un mondo popolato da stati sovrani in lotta per la supremazia mondiale l'esito è Auschwitz o, peggio, l'olocausto nucleare e la fine della specie umana. Non esiste un'utopia positiva per un'ideologia che si fonda sull'odio tra l'amico (il connazionale) e il nemico (lo straniero) e sulla produzione di micidiali armi per sterminare gli avversari. Nell'epoca dell'interdipendenza sociale planetaria, del mercato globale e della rete mondiale delle tecnologie dell'informazione, sostenere che i popoli nazionali debbano restare chiusi nei loro confini nazionali significa distruggere le basi materiali del benessere e della civiltà. Il nazionalismo di seconda generazione è l'ideologia che non può concepire un futuro per l'umanità, è una distopia.

La mia conclusione è pertanto che le ideologie del liberalismo, della democrazia e del socialismo che si ispirano agli ideali dell'umanesimo e dell'illuminismo possono abbracciare, se lo vorranno, l'ideale del progresso indicato con estrema chiarezza nel Manifesto di Ventotene. L'Unione europea sta già percorrendo questo cammino e lo stanno percorrendo i movimenti e i partiti ecologisti. Tuttavia, non mi faccio illusioni sulla difficoltà che si dovranno superare. Non si tratta solo degli interessi nazionali che impediscono ai governi di percepire con chiarezza cosa fare per il futuro del Pianeta, non sono meno importanti le resistenze ideologiche che oppongono gli intellettuali alla nuova 
idea di progresso. Faccio solo alcuni esempi tratti da studi recenti di sinceri umanisti e difensori degli ideali dell'illuminismo. Steven Pinker sostiene: «Molti scienziati sono ingenui quando entrano in discussioni di politica e diritto e propongono fallimentari progetti come un governo mondiale... o l'abbandono della Terra per colonizzare altri pianeti» (Pinker 2018: 390). Lo stesso Pinker, tuttavia, in altre pagine riporta osservazioni condivisibili, come questa: «Nulla vieta alla natura umana di impedire che una persona sia un fiero francese, Europeo e cittadino del mondo nello stesso tempo» (p. 450). Ma si può essere cittadini del mondo in stati dotati di armi per lo sterminio di massa e disposti a scatenare una guerra? È compatibile questa condizione umana con gli ideali umanistici che Pinker difende con tanta energia, precisione e competenza? Un secondo esempio è rappresentato dal filosofo Kwame A. Appiah che nel suo saggio Cosmopolitanism, scrive: «Vi sono pochi politici cosmopolitici che desiderano un governo mondiale. Ma il cosmopolitismo che sto difendendo valuta una varietà di istituzioni politiche, ammesso, naturalmente, che ogni stato garantisca a ogni individuo quello che lui o lei desiderano. Uno stato globale avrebbe almeno tre ovvi problemi. Esso accumulerebbe un potere incontrollabile, che potrebbe venir usato per produrre molte sofferenze; sarebbe spesso non responsabile verso le esigenze locali; e ridurrebbe quasi certamente la varietà delle istituzioni dalle quali noi tutti possiamo imparare qualcosa» (Appiah 2007: 163). Eppure Appiah vive negli Stati Uniti, una federazione con cinquanta stati membri che il governo federale, per ora, non ha ancora privato della loro indipendenza dal governo centrale. Inoltre, non ha soppresso le libertà fondamentali dei cittadini di una unione di stati che ha come motto: Unity in Diversity. Vi è poi l'esempio dell'Unione europea, dove non si può certo affermare che i poteri sovranazionali dell'Unione soffochino la libertà dei cittadini europei, sebbene le discussioni sui rapporti tra governi nazionali e poteri dell'Unione siano spesso aspre e interminabili. Infine, Tzvetan Todorov afferma che gli stati esistenti nell'Unione europea difendono un'idea di civiltà che ingloba i valori fondamentali e rifiutano la barbarie che li nega. Ma aggiunge che «l'individuo non può rivendicare i suoi diritti che nella misura in cui uno stato li garantisca e, in caso di necessità, intervenga per difenderli. Ci si può sentire nell'animo profondamente cosmopoliti, non si è mai cittadini del mondo» (Todorov 2008: 126). Eppure Todorov condanna fermamente il nazifascismo con tutti i suoi orrori. Perché non chiedersi per quali ragioni l'Unione europea oggi è 
così differente dall'Europa che ha generato quei regimi? Todorov non lo spiega. Il cosmopolitismo non è conciliabile, se non nei sogni, con un mondo in cui i governi nazionali praticano la politica di potenza e difendono i princìpi della supremazia nazionale sugli altri popoli. La prova tangibile che la vecchia concezione dei rapporti tra i popoli nazionali è tramontata in Europa è che alcuni poteri - non tutti ovviamente - che potrebbero causare conflitti violenti tra stati sono stati affidati a un'Unione sovranazionale.

In conclusione, nonostante tutte le difficoltà e le obiezioni che ho qui ricordato, sono convinto che la lotta politica per garantire all'umanità un futuro di pace, di cooperazione economica e politica per la salvezza del Pianeta, possa essere vinta. La prima difesa della vita è la volontà di vivere. I giovani che oggi protestano insieme in tutti i continenti dimostrano che gli ideali dell'umanesimo e dell'illuminismo sono radicati nella cultura mondiale contemporanea. Sono questi ideali a unire i giovani nella rivolta contro l'immobilismo dei governi nazionali.

\section{BIBLIOGRAFIA}

Albertini M., 1999, Nazionalismo e federalismo, Bologna, Il Mulino.

Appiah K. A., 2007, Cosmopolitanism. Ethics in a World of Strangers, London, Penguin Books.

Carr E.H., 1966, Sei lezioni sulla storia, Torino, Einaudi; trad. it. di What is History?, London, Macmillan, 1961.

Carr E.H., 1981, The Twenty Year's Crisis, 1919-1939. An Introduction to the Study of International Relations, London Macmillan.

De Ruggero G., 1962, Storia del liberalismo europeo, Milano, Feltrinelli.

Gardiner S., 2011, A Perfect Moral Storm. The Ethical Tragedy of Climate Change, Oxford, Oxford University Press.

Jonas H., 1993, Il principio responsabilità, Torino, Einaudi; trad. it. di Das Prinzip Verantwortung, Frankfurt am Main, Insel Verlag.

Magnani L., 2012, Filosofia della violenza, Genova, Il Melangolo; trad. it. di Understanding Violence, Berlin, Springer, 2011.

Mannheim K., 1957, Ideologia e utopia, Bologna, Il Mulino; trad. it di Ideologie und Utopie, Cohen, Bonn, 1929.

Montani G., 2019a, Ideologia, economia e politica. Il federalismo sovranazionale come pensiero emergente, Pavia University Press, 2019.

Montani G., 2019b, Supranational Political Economy. The Globalisation of the StateMarket Relationship, London and New York, Routledge.

Morin E., 2018, Conoscenza, ignoranza, mistero, Milano, Raffaello Cortina; trad. it. di Connaissance, ignorance, mystère, Paris, Fayard. 
Pinker S., 2018, Enlightment Now. The Case for Reason, Science, Humanism and Progress, London, Penguin Books.

Ricour P., 2017, Philosophie, étique et politique. Entretiens et dialogues, Paris, Seuil.

Searle J., 2006, La costruzione della realtà sociale, Torino, Einaudi; trad. it. di The Construction of Social Reality, New York, The Free Press, 1995.

Searle J., 2010, Creare il mondo sociale. La struttura della civiltà umana, Milano, Raffaello Cortina; trad. it. di Making the Social World. The Structure of Human Civilization, Oxford, Oxford University Press, 2010.

Todorov T., 2008, La peur des barbares. Au-delà du choc des civilisations, Paris, Laffont. von Right G.H., 1976, "Il determinismo e lo studio dell'uomo" in Simili R. (a cura di), La spiegazione storica. Prospettive recenti nella filosofia analitica, Parma, Pratiche Editrice, 1984.

Weber M., 1966, Il lavoro intellettuale come professione. Due saggi, Torino, Einaudi; trad. it. di Politik als Beruf, Wissenschaft als Beruf, Berlin, Duncker \& Humblot, 1921. 